\title{
Low radioactivity argon and SiPMs at cryogenic temperatures for the next generation dark matter searches
}

\section{Walter M. Bonivento*†}

Istituto Nazionale di Fisica Nucleare, Sezione di Cagliari, Cittadella Universitaria di Monserrato, Monserrato (CA), Italy

E-mail: walter.boniventodca.infn.it

\begin{abstract}
The next generation experiment for argon dark matter searches, DarkSide-20k (DS-20k) at Laboratori Nazionali del Gran Sasso (LNGS) Italy, aims at an exposure of $100 \mathrm{t}$, with a sensitivity in terms of exclusion limits for spin independent interaction of weakly interacting massive particles (WIMPs) of $3 \times 10^{-48} \mathrm{~cm}^{2}$ at $200 \mathrm{GeV}$ of mass, and is expected to come into operations in 2021. Two crucial technologies are being developed for this experiment, i.e. the extraction and purification of low-radioactivity argon from deep underground and a new generation of silicon photo-multpliers (SiPMs), developed on purpose for this project. The same worldwide collaboration is also pursuing studies towards a possible future next-to-next generation experiment, aiming at an exposure of $1000 \mathrm{t} y$, with sensitivity possibly reaching the neutrino floor.
\end{abstract}

The European Physical Society Conference on High Energy Physics

5-12 July, 2017

Venice

* Speaker.

${ }^{\dagger}$ on behalf of the DarkSide-20k Collaboration 


\section{Direct Dark Matter search}

Dark Matter (DM) is one of the most elusive mysteries of science. Indirect evidence for dark matter is overwhelming, though mostly of gravitational nature, and include studies of rotation curves of galaxies and clusters, gravitational lensing and cosmic microwave background.

Physics beyond what is already known experimentally, summarised by the Standard Model (SM) of Particle Physics and by the framework of General Relativity, is required to explain it. DM particles ought to be neutral, non-baryonic and weakly interacting to be consistent with observations and cosmological constraints. Unfortunately, so far, no convincing evidence of a particle physics nature of the DM has been found either directly or indirectly.

Weakly Interacting Massive Particles (WIMP) that are thermal relics of the Big Bang are among the most interesting DM candidates. These particles are expected to undergo collisions with atomic electrons or nuclei of matter, depending on their mass, and over the last twenty years various detectors have been used to detect it, the most remarkable ones being with solid state and noble liquid targets. The mass region between few $10 \mathrm{GeV}$ and few $\mathrm{TeV}$ is favourite by cosmological constraints.

For WIMP masses above few GeV, detectors with xenon targets are now at the forefront of the research, with the PANDA-X [1] and the XENON1T [2] experiments leading the race with the best cross section exclusion limit for spin independent interactions, i.e. $1-2 \times 10^{-46} \mathrm{~cm}^{2}$ at $200 \mathrm{GeV}$ of mass.

Concerning liquid argon detectors, three experiments are currently running, i.e. DarkSide50 (DS-50) [3] at Laboratori Nazionali del Gran Sasso (LNGS) Italy, ArDM [4] at Laboratorio Subterraneo de Canfranc (LSC), Spain and DEAP-3600 [5] at SNOLAB, Canada, the latter holding the best upper limit, of $2 \times 10^{-44} \mathrm{~cm}^{2}$ at $200 \mathrm{GeV}$ of mass. Very recently the above mentioned liquid argon detector collaborations decided to formally merge into a Global Argon Dark Matter Collaboration, with the first goal being a next generation effort, i.e. the DarkSide-20k (DS-20k) experiment at LNGS [6], that aims at an exposure of $100 \mathrm{t} y$, with a sensitivity in terms of exclusion limits of $3 \times 10^{-48} \mathrm{~cm}^{2}$ at $200 \mathrm{GeV}$ of mass. This reach is comparable with the xenon-based detectors LZ at SURF, USA [7] and XENONnT [8] at LNGS, Italy. DS-20k has recently been approved by both LNGS and INFN Committees.

The Global Argon Dark Matter Collaboration is also pursuing studies towards a possible future next-to-next generation upgrade of the DS-20k experiment, aiming at an exposure of $1000 \mathrm{t} y$, with sensitivity possibly reaching the neutrino floor.

Two crucial technologies are being developed for this experiment, i.e. the extraction and purification of low-radioactivity argon from deep underground and a new generation of silicon photo-multpliers (SiPMs), developed on purpose for this project.

\section{The DS-20k detector}

The core of the DS-20k experiment is an octagonal double-phase liquid argon (LAr) TPC with an active (fiducial) mass of $23 \mathrm{t}(20 \mathrm{t}$ ) low-radioactivity argon, with a height of $239 \mathrm{~cm}$ and a distance between parallel walls of $290 \mathrm{~cm}$. The low recoil energies and cross sections targeted with DS-20k represent an enormous experimental challenge, especially in the face of daunting back- 
grounds from electron recoil interactions and from neutrons that mimic the nuclear recoil signature of WIMPs. To meet its challenge, the cryostat will be contained into a cylinder-shaped transparent acrylic vessel capped off at its two ends by two small-radius spherical domes. Preliminary estimates indicate a radius of about $3 \mathrm{~m}$ and a height of from $6 \mathrm{~m}$ to $7 \mathrm{~m}$. This vessel is going to be filled with boron-loaded liquid scintillator, serving as an active neutron veto (LSV). This vessel will sit inside a $15 \mathrm{~m}$ diameter $14.65 \mathrm{~m}$ tall stainless steel tank, filled with ultra-pure water, functioning as an active muon veto (WCV). Energy deposits in the LAr target result in a characteristic production of excited and ionised argon atoms, according to the underlying process of a recoiling electron or nucleus. Excited argon atoms, which can also be produced by recombining ionisation charge, lead to an efficient formation of argon excimers decaying via the emission of scintillation light containing two components with different time constants of emission. Both components combine together to yield an instant light signal S1. Due to the deep UV nature (around $128 \mathrm{~nm}$ ) of this scintillation light, a thin layer of wavelength shifter must cover all exposed surfaces. Ionisation electrons escaping recombination are drifted by an applied electric field to the top of the LAr, where a stronger applied field extracts the electrons into the gas pocket above the liquid. Here the strong field accelerates the electrons, enough for them to excite the argon gas, producing a secondary scintillation signal S2, proportional to the ionisation charge. Photosensors placed behind wavelength shifter coated windows at the top and bottom of the TPC, read out both scintillation signals in each event. S1 is used for energy determination, as well as for pulse shape discrimination (PSD) [9]. PSD allows to suppress electron recoil backgrounds compared to the signal nuclear recoil by a huge factor in argon, in excess of $10^{9}$ [6], and it is derived from the ratio of the prompt and delayed light fractions. S2 is used for energy and 3D position measurement of the event: the vertical coordinate from the drift time between S1 and S2, and the horizontal coordinates from the light pattern in the top photosensors.

\section{The new SiPMs for DS-20k}

The DS-20k TPC will be instrumented with a new kind of photosensors, arrays of SiPMs, arranged in assemblies called photodetector modules (PDMs), consisting of a large tile of 24 rectangular SiPMs covering an area of $25 \mathrm{~cm}^{2}$ operating as a single detector. Each PDM has an area comparable to that of a $3^{\prime \prime}$ photomultiplier tube (PMT), with the LAr TPC containing 5210 PDMs in total. Substantial effort was put in the developments of this technology, since SiPMs promise a higher effective quantum efficiency, higher reliability at LAr temperature, and a much higher radio-purity than PMTs. All of these properties are crucial for DS-20k: PSD, in LAr the most important mechanism for background discrimination, depends critically on the light yield, while the large material budget of PMTs is often the limiting factor for neutron- and gamma-induced backgrounds. The LAr TPC will be equipped with arrays of SiPMs, totalling $14 \mathrm{~m}^{2}$ in area.

Fondazione Bruno Kessler (FBK) [10] has developed the SiPM technology with high density single photon avalanche diodes that have peak efficiency in the near ultra-violet $(410 \mathrm{~nm})$. The SiPM layout and the front-end electronics have been designed to achieve a signal to noise ratio (SNR), defined here as the ratio between the amplitude of the single photo-electron signal and the baseline noise, better than 10. At this noise level, the rate of baseline fluctuations misidentified as signals is well below the intrinsic dark rate of the SiPMs and the detector can operate as a 
single photon counter. In addition, the high bandwidth front-end electronics maintain the timing characteristics of the SiPM signals and allow for timing resolution better than $20 \mathrm{~ns}$.

The SiPM tile is mated to a board housing the front-end electronics. The 24 SiPMs tile is subdivided into $6 \mathrm{~cm}^{2}$ quadrants, and each quadrant is read out with an independent low-noise trans-impedance amplifier (TIA). Two of these SiPMs tiles, actually made of squared $1 \mathrm{~cm}^{2} \mathrm{SiPMs}$ from a test production, underwent laboratory testing and they were shown to satisfy the DS-20k requirements i.e. Photon Detection Efficiency in excess of 40\%, Dark Count Rate lower than $0.1 \mathrm{~Hz} / \mathrm{mm}^{2}$ at LAr temperature and SNR in excess of 10, as discussed in [12, 13].

\section{The low radioactivity argon}

The isotope with atomic mass of 39 (most of the argon has atomic number 40 ), a $\beta$-emitter with $\mathrm{Q}=565 \mathrm{keV}, \mathrm{T}_{1 / 2}=269 \mathrm{y}$, is produced by cosmic rays in the atmosphere at the level of $1 \mathrm{~Bq}$ per kg or 0.112 part per million (ppm) per litre of argon.

PSD in liquid argon provides strong suppression of this background above a given threshold, this last depending both on light yield (i.e. the number of detected photo-electrons per $\mathrm{keV}$ of deposited energy) and on the amount of background. For instance, for the DS-20k experiment, the PSD threshold is expected to be around $8 \mathrm{keV}$ of e- recoil energy. Below this threshold the ${ }^{39} \mathrm{Ar}$ constitutes an irreducible background for DM searches. For double phase detectors above the tonne scale, the ${ }^{39} \mathrm{Ar}$ background would anyway prevent operation due to pile-up in the detector, since for these large detectors the drift time becomes of order of ms.

The DS-50 experiment at LNGS is the first detector running with an argon target low in ${ }^{39} \mathrm{Ar}$, i.e. with a radioactivity less than one thousandth of that of atmospheric argon [3]. For dark matter searches, low-radioactivity argon is of interest for DS-20k and its possible upgrade but also for further progress of the DEAP-3600 detector at SNOLAB.

In the mid 2000s [11] it was found that argon present in $\mathrm{CO}_{2}$ gas wells from the earth mantle has a much-reduced content of this isotope, at the level of less than 1 per mil of the atmospheric one. A first use case for this argon was the DS-50 experiment at LNGS that used about $153 \mathrm{~kg}$ of the argon, named thereafter underground argon (UAr), extracted and purified with a five-year effort at Cortez, Colorado.

The DS-20k experiment will make use of $30 \mathrm{t}$ of this low radioactivity argon extracted by the mine at Cortez, and purified using two new plants in series, one named Urania located at the extraction site and another one, named Aria, located in the Carbosulcis mine of Nuraxi-Figus in Sardinia (Italy), a $350 \mathrm{~m}$ tall cryogenic isotopic distillation column.

Urania will provide argon at the rate of $100 \mathrm{~kg} /$ day with a purity of $99.9 \%$, starting from $95 \%$ $\mathrm{CO}_{2}$ and $440 \mathrm{ppm}$ of UAr with $90 \%$ recovery, and subsequently Aria will further purify it to achieve contaminant levels below the part per billion ( $\mathrm{ppb}$ ), i.e. to make it detector-grade. The Urania plant is under advanced design and, according to the schedule, installation should start early next year.

The Aria plant, that was designed as cryogenic isotopic distillation plant for the abatement of ${ }^{39} \mathrm{Ar}$, is capable to suppress the radioactive isotope by another factor of ten per pass. Aria consists of two steps: Seruci-I, i.e. a $30 \mathrm{~cm}$ diameter columns, that is already funded and is capable of isotopic distillation with a rate of $10 \mathrm{~kg} / \mathrm{day}$ and Seruci-II, a $1.5 \mathrm{~m}$ diameter column, that is however not yet funded, and would be capable of isotopic distillation at a rate of $150 \mathrm{~kg} /$ day. At present about one 
half of the Seruci-I modules are already produced and tested at CERN, Switzerland, for leakage. The modules are ready to be shipped to Sardinia for installation inside the Seruci well, that is in advanced state of refurbishing.

Seruci-I will be used for DS-20k only for removal of chemical impurities to make the UAr detector-grade with two passes of chemical purification at a rate of 1 t/day with $85 \%$ recovery. Indeed, the inlet purity required by DS-20k getters is of the order $0.25-1 \mathrm{ppm}$. The isotopic cryogenic distillation feature will only be used for testing purposes, to prepare the grounds for Seruci-II, if the tests of Seruci-I were successful.

Both the DS-20k experiment and the two plants are international projects funded by INFN, by Italian regional fundings, and by other agencies, such as the National Science Foundation, USA.

\section{Conclusions}

We have discussed two crucial technologies that are being developed for the DS-20k experiment and future upgrades, i.e. the extraction and purification of low-radioactivity argon from deep underground and a new generation of silicon photo-multpliers (SiPMs). The DS-20k experiment is expected to be operative starting from 2021.

\section{References}

[1] X.Cui et al., Dark Matter Results From 54-Ton-Day Exposure of PandaX-II Experiment, arXiv:1708.06917 (2017)

[2] E.Aprile et al., First Dark Matter Search Results from the XENONIT Experiment, arXiv: 1705.06655 (2017)

[3] P.Agnes et al., Phys.Rev. D 93 (2016) no.8, 081101, Addendum: Phys.Rev. D 95 (2017) no.6, 069901

[4] J.Calvo et al., JCAP 1703 (2017) no.03, 003

[5] P.A.Amaudruz et al., First results from the DEAP-3600 dark matter search with argon at SNOLAB, arXiv:1707.08042 (2017)

[6] C.E.Aalseth et al., DarkSide-20k: A 20 Tonne Two-Phase LAr TPC for Direct Dark Matter Detection at LNGS, arXiv:1707.08145 (2017)

[7] B.J.Mount et al., LUX-ZEPLIN (LZ) Technical Design Report, LBNL-1007256, arXiv:1703.09144 (2017)

[8] E.Aprile et al., JCAP 1604 (2016) no.04, 027

[9] M.G. Boulay and A.Hime, Astropart. Phys. 25 (2006) 179-182

[10] Fondazione Bruno Kessler, Via Santa Croce 77, 38122 Trento, Italy

[11] J.Xu et al., Astropart. Phys. 66 (2015) 53-60

[12] M.D'Incecco et al., Development of a novel single-channel, 24 cm2, SiPM-based, cryogenic photodetector, arXiv:1706.04220v2 (2017)

[13] M.D'Incecco et al., Development of a very low-noise cryogenic pre-amplifier for large-area SiPM devices, arXiv:1706.04213 (2017) 